\title{
„Die zweigriffige Baumsäge“. Ein biokybernetisch-medienökologisches Experimentalsystem
}

\author{
Martin Dornberg und Daniel Fetzner
}

\section{Zusammenfassung}

Das im Rahmen psychosomatischer und neurologischer Grundlagenforschung an der Uniklinik Heidelberg entwickelte Experimentalsystem der ,zweigriffigen Baumsäge" kann als bio-kybernetisches Experimentalsystem erster Stunde bezeichnet werden. Der Psychosomatiker und Philosoph Martin Dornberg und der Medienkünstler Daniel Fetzner diskutieren in einem Gespräch Kontexte und Folgen der um den Heidelberger Psychosomatiker Viktor v. Weizsäcker in den 1940er und 1950er Jahren durchgeführten medizinischen Experimente - in den Bereichen von Medizin und Psychotherapie einerseits, aber auch in den Technik- und Medienwissenschaften, der Kybernetik und der Medienkunst. Herausgearbeitet werden Querbezüge zu den von den Autoren durchgeführten künstlerisch-philosophischen Forschungen in Form von Installationen, Performances und interaktiven Webdokumentationen.

M. Dornberg $(\bowtie)$

Philosophisches Seminar und Zentrum für Anthropologie und Gender Studies, Universität Freiburg, Freiburg, Deutschland

E-Mail: dornberg.freiburg@t-online.de

D. Fetzner

Hochschule Offenburg, Fakultät Medien, Offenburg, Deutschland E-Mail: daniel.fetzner@hs-offenburg.de

(C) Springer Fachmedien Wiesbaden GmbH, ein Teil von Springer Nature 2020

B. Ochsner et al. (Hrsg.), Affizierungs- und Teilhabeprozesse zwischen

Organismen und Maschinen, Technikzukünfte, Wissenschaft

und Gesellschaft / Futures of Technology, Science and Society,

https://doi.org/10.1007/978-3-658-27164-0_7 


\section{Schlüsselwörter}

Verkörperung · Künstlerische Forschung · Medienökologie · Transmedialität ·

Zwischenleiblichkeit

Experimente zur interpersonellen Zweierbeziehung, die im Kontext der von Viktor v. Weizsäcker begründeten Gestaltkreistheorie (Weizsäcker 1973) entstanden sind, haben am Universitätsklinikum Heidelberg eine lange Tradition in Forschung und Lehre. So entwickelten dort der Neurologe Paul Christian und die Sozialarbeiterin Renate Haas (1949) das Modell der zweigriffigen Baumsäge zur experimentellen Überprüfung der Merkmale dyadischer Beziehungsstrukturen, die sie als Bipersonalität bezeichneten (Dornberg 2013). Die Ergebnisse der Untersuchungen waren beschreibend und lieferten unter anderem die Konzepte der „Selbstverborgenheit im Tun“ (Christian und Haas 1949, S. 11) und des „Handgemenges“ (ebd., S. 67) und bereicherten auch die neurologisch-rehabilitationsmedizinische Grundlagenforschung.

Zwei Personen arbeiten an einer zweigriffigen Baumsäge. Ihre Bewegungen werden von einer Apparatur registriert, und das subjektive Erleben der Beteiligten wird laufend abgefragt, aber auch die quantitative Leistung wird erfasst: Form, Richtung, Größe, Dauer der Bewegungen der Sägestange, die von den Partnern aufgewendeten Kräfte bzw. Arbeit sowie deren jeweilige Selbst- und Fremdwahrnehmung.

Aus diesen Experimenten können eine Fülle von Schlussfolgerungen über gelingende Interaktionen aufeinander bezogener Menschen und auch über aufeinander bezogene Lebens- und Interaktionsprozesse abgeleitet werden. Sie zeigen, dass Leistungen (wie das Baumsägen) durch gelingende Abstimmungsprozesse untereinander entstehen: zwischen den beteiligten menschlichen und nichtmenschlichen/dinglichen Akteuren und durch die Art der Aufgabe. Rückmeldeprozesse finden bei allen Beteiligten gleichzeitig durch den Gesamtprozess, die beteiligten Aktanten (z. B. Holz/Säge/Erkrankungen der Beteiligten) sowie die realisierten Leistungen statt. Die einzelnen Individuen, ihre Körper und Aktionen, verschmelzen mit dem Gesamtgeschehen und der Leistung zu einem neuen ,überpersonalen“ Ganzen: zu einer Art Gesamtkörper, den man in Anlehnung an eine Formulierung Klaus Theweleits ,[d]ritter Körper“ nennen kann (Theweleit 2007). 
Die Experimente mit der zweigriffigen Baumsäge wurden auch mit körperlich erkrankten Patienten durchgeführt. Besonders überraschend waren dabei die Experimente, die das gemeinsame Handeln von einem Gesunden mit einem motorisch gestörten Partner etwa mit extrapyramidaler Bewegungsstörung/ Morbus Parkinson untersuchten:

\begin{abstract}
„Der gesunde Partner im Sägeversuch setzt nicht um jeden Preis sein bzw. das normale Verhalten durch, sondern opfert spontan die normale Arbeitsform zugunsten einer anderen. Meist übernimmt er spontan einige Glieder des Arbeitsvorgangs und überlässt dem Kranken die einfacheren Glieder. So zieht z. B. der extrapyramidal Bewegungsgestörte [d. h. der Mensch mit Morbus Parkinson, M.D./D.F.] bei einer rhythmisch alternierenden Arbeit das Arbeitsobjekt nur taktmäßig an sich und überlässt dem Gesunden Lenkung und Rückführung oder er übernimmt nur die Abbremsung an den Wendepunkten und überlässt alles andere dem gesunden Partner. Die Arbeitsform wird so weniger nuancenreich und in gewissem Sinne auch primitiver, aber der Arbeitsbezug blieb erhalten." (Christian 1989, S. 265f.)
\end{abstract}

Die Experimente zum Sägevorgang mit Gesunden und Kranken zeigen, dass sich beide in der Zusammenarbeit so aufeinander einzustellen vermögen, dass im Zusammenspiel die pathologische Funktion bestmöglich ausgeglichen wird und dadurch fast eine normale Leistungsfähigkeit wie bei zwei gesunden Sägepartnern erreicht werden kann: Dabei überbrückt der Gesunde die Störung nicht durch zusätzlichen Arbeitsaufwand, sondern annihiliert sie eher durch Umformung. Diese Erfahrungen wurden vor allem in der Rehabilitation neurologisch Erkrankter, aber auch für die Aus- und Weiterbildung von Medizinstudenten und Ärzten wirksam. Das Modell der zweigriffigen Baumsäge stellt nämlich eine innovative Möglichkeit dar, die Zweierbeziehung als Grundlage ärztlicher Kommunikation und der ärztlichen Tätigkeit systematisch zu reflektieren und die dazu gehörigen Wahrnehmungs- und Interaktionsvorgänge kognitiv und emotional erlebbar zu machen. Durch die zeitgleiche Erhebung von nicht wahrnehmbaren physiologischen Reaktionen und subjektiver Wahrnehmung wird eine Situation geschaffen, in der das Interaktionsgeschehen in besonderer Weise multimodal erfahrbar und komplexe Vorgänge polysystemisch beschreibbar werden. Für die weitgehend naturwissenschaftlich ausgebildeten Studenten und Ärzte wird so ein Kontakt mit ergänzenden qualitativen Methoden in einem Rahmen ermöglicht, der Bedeutung und Nutzen solcher, weicher' Daten und die Entstehung interaktioneller und verkörpernder Emergenz im medizinischen Kontext direkt veranschaulicht. 
Im folgenden Gespräch sprechen Daniel Fetzner und Martin Dornberg über die Bedeutung der Baumsägeexperimente als biokybernetisches und medienökologisches Experimentalsystem im Kontext ihrer künstlerischen Forschung. ${ }^{1}$

Daniel Fetzner (DF): Mit dem Experimentalsystem der „Baumsäge“ verbinden wir sehr unterschiedliche Vorgeschichten. Den konkreten Versuchsaufbau habe ich über Dich 2009 bei unserem Workshop in Furtwangen kennengelernt. Das Pendelexperiment von Christian und Haas (1949) war mir ja bereits über Stefan Riegers Kybernetische Anthropologie (2003) bekannt und spielte in meinem Projekt ,fogpatch“2 (2007-2010) zu Max Bense eine maßgebliche Rolle. An dem „Prinzip Baumsäge“ interessierte mich bei unserer Zusammenarbeit vor allem die Erweiterung um die Dimension der Translokalität (Fetzner 2009).

Martin Dornberg (MD): Aus meiner Sicht hat dein Bense-Projekt tatsächlich schon diesen Gedanken der Bio-Kybernetik durchexerziert und auf verschiedene Art und Weise entfaltet, mit dem Schauspieler, der an Elektroden angeschlossen ist oder dem IT-Pionier, der alias Bense über die Golden Gate Brücke läuft. ${ }^{3}$ Wie bist du eigentlich da darauf gekommen oder wie würdest du dieses Projekt auf die Baumsäge umlegen?

DF: Bense entwickelte 1951 mit seiner „Technischen Existenz“ (Bense 1998) eine Utopie der medialen Durchdringung des modernen Menschen mithilfe von computerisierter Technologie. Auf den Text bin ich während meiner Lehrtätigkeit an der Hochschule Furtwangen gestoßen. In der dortigen Betriebs- und Ausbildungslogik steckt dieses Technikversprechen unausgesprochen in allen

\footnotetext{
${ }^{1}$ Unsere Projekte selbst verstehen sich im Sinne Hans-Jörg Rheinbergers als Experimentalsysteme, die Beobachtungs- und Handlungssituationen schaffen, um transdisziplinär bestimmte Phänomene wie z. B. das der Zwischenleiblichkeit, der Verkörperung usf. beforschen wollen. Experimentalsysteme überschreiten oft die Grenzen einzelner Disziplinen und beforschen dabei auch sich selbst, ihre eigene Methodik, Prozessualität und Materialität. Experimentalsysteme sind also unterschiedlich dimensionierte, materialisierte Versuchsanordnungen, welche dazu geeignet sind, wissenschaftliche Fragen weiterzutreiben, Fragen und Antworten zu schaffen, sowie wissenschaftliche Theorie und Praxis voranzubringen (Rheinberger 2001). Dabei sind Theorie und Praxis, institutionelle und technische Aktanten und Forschung(sergebnisse) eng voneinander abhängig. Experimentalsysteme dürfen laut Rheinberger nicht zu eng, aber auch nicht zu weit sein und dürfen, ja müssen manchmal „unmögliche“ Fragen stellen, um (neue) Antworten hervorzubringen. Die Baumsägeexperimente stellen ein „typisches Experimentalsystem“ dar (Rheinberger, persönliche Mitteilung).

${ }^{2}$ http://fogpatch.de. Zugegriffen: 8. Juni 2018.

${ }^{3}$ Unter Beteiligung der Schauspieler Georg Hobmeier und Frank Dietrich.
} 
Ritzen und Poren - ohne dass dabei irgendjemand Bense gelesen hätte. ${ }^{4}$ Als Gestalter in Nachbarschaft mit Informatikern war ich konkret immer wieder über Notengebungen mit der absurden Frage nach der Berechenbarkeit bzw. Messbarkeit des Ästhetischen konfrontiert. Und da lag Benses Informationsästhetik als gescheiterte und durchaus komische Ambition der kybernetischen Moderne förmlich auf der Hand.

Als Grenzgänger zwischen den ,beiden Kulturen“ (Snow 1957) hat Max Bense sich schon zu Kriegszeiten mit technischen Körperphantasien befasst, was mich neugierig machte. Am Institut für Hochfrequenztechnik wollte er sich 1943 in Gestalt eines „Rasterkörpers“ von Berlin nach San Francisco beamen, um dort mit den Dingen in Interaktion zu treten. Das machte er dann dreißig Jahre später auf ganz eine andere, reale, physische Weise. Zusammen mit Studierenden in Furtwangen und Kairo haben wir von 2007 bis 2010 interaktive Projekte, Ausstellungen und Symposien organisiert, um Benses „Existenzmitteilung aus San Franzisko“ (1970) in Form von biokybernetischen Experimentalsystemen zu materialisieren. Das war ein sehr intensives Wechselspiel zwischen Philosophie und medial-ästhetischer Anwendung, in gewisser Weise mein zweiter Einstieg ${ }^{5}$ in die künstlerische Forschung und vor allem der Beginn unserer Zusammenarbeit.

Ein zentraler materialer Aktant in „fogpatch“ ist neben dem Nebel die Golden Gate Brücke in San Francisco, über die Bense 1969 zu Fuß gehen muss, weil er den Bus verpasst. Daraufhin bekommt er eine Nierenkolik, die er dann in seiner Existenzmitteilung transmedial faltet. Der Text kann so als eine Art bodily turn in Benses Philosophie gelesen werden. Die Bezugnahme zu der schwingenden Brücke und $\mathrm{zu}$ seiner eigenen Körperlichkeit als eine Art biokybernetisches Experimentalsystem löst in Benses Denken eine schwere Krise aus. Die existentielle Erfahrung setzt eine Transformation in Gang, die seine kybernetische Sicht der Dinge wendet und den Körper ins Zentrum des Geschehens rückt. Das haben wir 2008 auf der Tagung „Spuren“6 über die Performance „Seismic Body Signals" gezeigt ${ }^{7}$.

\footnotetext{
${ }^{4}$ Mit Ausnahme meines Informatikerkollegen Wolfgang Taube, dem ich hier für viele kritische Anregungen und Gespräche dankbar bin.

${ }^{5}$ Als meine (DF) erste künstlerische Forschung würde ich das Wanderkinoprojekt lüderitzcargo in Namibia 1995/1996 bezeichnen, siehe http://cargo.metaspace.de. Zugegriffen: 8. Juni 2018. ${ }^{6}$ http://www.metaspace.de/sub/spuren2008. Zugegriffen: 8. Juni 2018.

${ }^{7}$ http://fogpatch.de. Zugegriffen: 8. Juni 2018.
} 
Ich kann mich noch gut an eines unserer ersten Treffen in Deiner Praxis erinnern. Dort hast du auf einem Whiteboard eine Systemskizze aufgemalt, in der du diese Gefügebildung von Bense/Brücke/Text psychosomatisch, also über Verbindungen von Geist/Text und Dingen/Verkörperungen zu erklären versuchtest. So haben wir uns kennengelernt (Abb. 1).

MD: Was hat Bense aus deiner Sicht an der Biokybernetik interessiert? Ein Spieleinsatz von ihm geht in die Richtung der Berechenbarkeit des Ästhetischen und du sagst, er hat eine gewisse Entwicklung vom Mathematisch-Technischen zum Körperlicheren durchlaufen. Vielleicht ist das auch eine Brücke zur Frage der „künstlerischen Forschung“: das Ästhetische, das Affektive und solche Dinge wie ein „dritter Körper“ (Dornberg 2014), also etwas das sich zwischen zwei Interaktionspartnern, ÄrztInnen und PatientInnen oder zwischen zwei ImprovisationsmusikerInnen entwickelt, ist das berechenbar? Wie gestaltet sich diese Spannung, jene transmediale Beziehung zwischen Berechenbarkeit und Erfahrung? Wie hat Bense das ursprünglich gedacht und wie hat sich das deiner Sicht nach entwickelt?

DF: Der „dritte Körper“ ist bei Bense selbsttherapeutisch über den Text der „Existenzmitteilung aus San Franzisko“ entstanden. Körper-Bense und Körper-Brücke begegnen sich als biokybernetisches System - , baumsägeartig “ und produzieren einen Textkörper, der im Stil der konkreten Poesie so verfasst ist, dass es ein nicht nur menschengedachter Korpus ist oder vorgibt zu sein. Dort

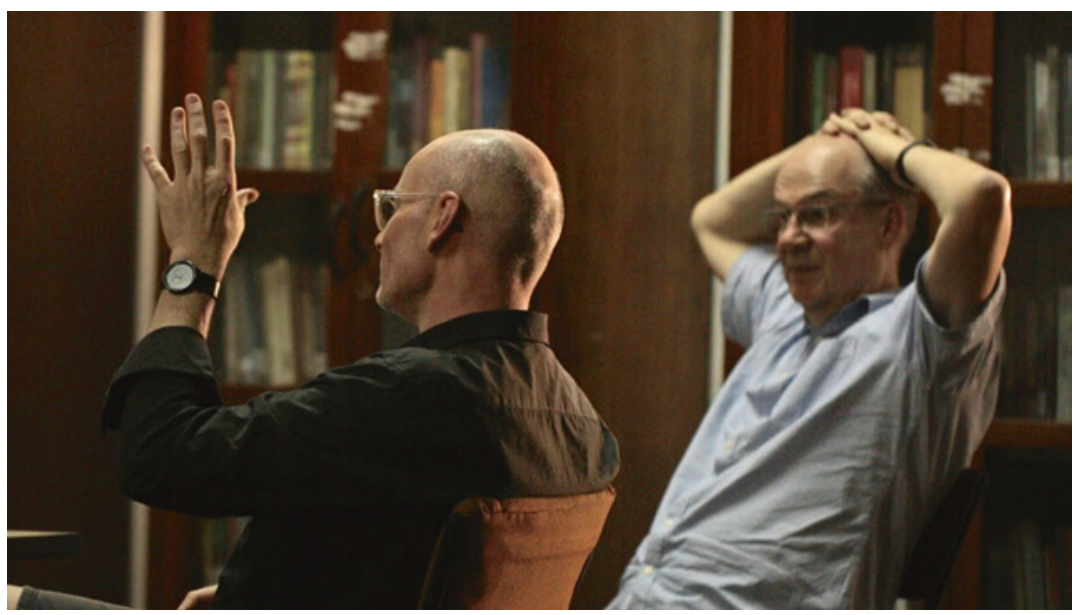

Abb. 1 Daniel Fetzner und Martin Dornberg, Fotograf: Adrian Schwartz 
erleben wir auch eine sehr starke Handlungsmacht von Qualitäten, Ereignissen oder Dingen. Wie der Farbe ,gelb‘ zum Beispiel, konkret maisgelb, oder den „Dummen-aus-dem-Bus-Blicken“ (Bense 1970, S. 9).

MD: Oder dem Nierenstein.

DF: Genau, der bringt die „Existenzmitteilung“ buchstäblich ins Rollen. Bense macht im materialistischen bzw. materialbezogenen Vorgriff die Dinge und die Attribute der Dinge schon sehr wirkmächtig und er arbeitet bereits 1970 an der Auflösung der Subjekt-Objekt-Dichotomie. Er hat das auch in anderen Arbeiten verfolgt. Nur wenige Monate nach der Existenzmitteilung produzierte er einen Text über das Autofahren. ${ }^{8}$ Da geht es ebenso um die Gefügebildung von Mensch und Maschine, die eine poststrukturalistisch gesprochen ,Ununterscheidbarkeitszone "9 eingehen, die sich jedem Kalkül entzieht. Es ist nicht mehr klar, wer fährt oder wer lenkt. Weder die Maschine noch der Mensch sind nunmehr autonom (Fetzner 2018). Dieses agencement (Deleuze) oder meshwork (Ingold) spielt sich in dem Interplay von Bense mit der Brücke ab, aber auch zwischen dem Auto und dem Menschen während des Fahrens. Zugleich ist die „Naht“ (Bense 1998, S. 291) zwischen technischer Existenz und technischem Objekt heute würde man von Interface sprechen - performativ und verkörpernd gedacht: das Abschreiten der Brücke und das Autofahren.

MD: Eigentlich gibt es zwischen der Berechenbarkeit und dem Rationalismus einerseits und dem Menschlichen andererseits für Bense keine Grenze. Das ist auch sein Affront gegen Beuys gewesen, dass er gesagt hat, Beuys ist viel zu affektiv, viel zu mythisch. Ich glaube, dass man Bense wirklich so verstehen könnte, dass er es sich in dieser Phase immer wieder zur Aufgabe gemacht hat, das Lebendige am Menschen in seiner ,technischen Existenz" berechenbar zu machen. Aber berechenbar heißt hier eben nicht , abstrakt" in einer reduzierten Art von Mathematik oder in einer einseitigen Art von Kybernetik, sondern berechenbar in einer Art ästhetischer Weise. Wo das Verständnis z. B. einer Lichtschaltung, die natürlich ganz klar berechenbar ist, bis hin zu konkreter Poesie und intensiven menschlichen Erfahrungen z. B. einer Erkrankung oder einer Liebesbeziehung mit immer komplexeren Formen von ,Berechenbarkeit" korrespondiert. Berechenbarkeit würde dann heißen, dass man in dem Geist oder dem Körper der

\footnotetext{
${ }^{8}$ „Indem es fährt, gewinnt das Auto den Rang eines Ortes, einer Linie, einer Naht gewissermaßen, an der Welt und Bewusstsein beständig zusammenstoßen; man könnte auch sagen: an der Sein und Denken zusammenstoßen." (Bense 1998 Band 4, S. 291).

${ }^{9}$ Zentraler Begriff von Deleuze und Guattari. Vgl. Deleuze und Guattar 1992; Deleuze 2000, 2016.
} 
,Rechnung' wie beheimatet sein müsste, also darauf bezogen, medial und ganzheitlich.

DF: Der antiaffektive Affekt bei Bense contra Beuys wurzelt in seiner Faschismuskritik. Bense war empfindlich gegen nebulös-mythische Inszenierungen, wie sie Beuys durchaus provozierend und mit anti-modernistischem Kalkül betrieben hat. Natürlich waren beide politisch links und es gab auch viele Überschneidungspunkte der Streithähne, aber in der legendären TV-Diskussion von 1970 hat Bense seine rationale Seite stark gemacht, um einen Standpunkt gegen Beuys einzunehmen. Das war nur wenige Monate nach dem Erlebnis seiner Nierenkolik und man kann diese Diskussion sehr gut als Teil des oben beschriebenen bodily turn verstehen. Beuys bringt die mathematische Kybernetik seines Kontrahenten ${ }^{10}$ hier kräftig ins Wanken. Du und ich sehen heute beide Ansätze ohne Probleme als biokybernetische an.

MD: Wenn ich unsere Projekte (Dornberg und Fetzner 2017) unter dieser Perspektive anschaue, würde ich da fast eine Art Bense'sche Handschrift sehen. Sie sind zum Teil sehr technisch, aber konzipieren Technik nicht als Selbstzweck, sondern betonen die Mensch-Maschine Interaktion, es sind Projekte technischer Existenz (Bense 1998). Die 360 Videos, die ein Interakteur steuert oder bedient, sei es mit der Brille oder mit der Maus (Fetzner 2017), unsere Skype-Performances oder auch das Bense-Projekt thematisieren durchgehend Hybridisierungen von Mensch und Technik. Wir arbeiten immer viel mit Algorithmen und in unseren Projekten wird zwischen den menschlichen, den dinglichen und den technischen Anteilen keine große Grenze gezogen.

DF: Alles sozusagen in der „Naht“ des Interaktionellen. Zusätzlich beinhalten diese Gefügebildungen translokale Momente. In unseren Projekten geht es darum, Räume miteinander in Verbindung zu bringen und die entstandene Relation auch zu verorten. Das Dialogische oder Triadische fand ich als Architekt immer im Hinblick auf konkrete Ortsbezüge spannend. Aber zurück zur Baumsäge: Wo taucht sie eigentlich in deinem Denken auf?

MD: Der Beginn meiner Beschäftigung mit der Baumsäge ist schnell benannt. Sie geht auf meine gute Bekanntschaft zu Thure von Uexküll, einem führenden Psychosomatiker zurück (Uexküll und Wesiack 1998). Einmal kam er zu mir und meinte, eigentlich müsste man bei den Ausbildungskursen für angehende

\footnotetext{
${ }^{10}$ Beide sprechen hier genüsslich von „Gegenerschaft“. Siehe Joseph Beuys - Künstler und Gesellschaft. Mitschnitt einer Fernsehdiskussion mit Joseph Beys, Max Bill, Arnold Gehlen und Max Bense. https://www.youtube.com/watch?v=SXPoAaBTPy8. Zugegriffen: 20. März 2018.
} 
PsychotherapeutInnen und PsychosomatikerInnen, eigentlich für jeden Arzt, gemeinsam sägen. Er hat uns mit diesen Baumsäge-Experimenten bekannt gemacht, die in den 1940er, 1950er Jahren in der Neurorehabilitation, in der Neurologie und der Inneren Medizin an der Universitätsklinik in Heidelberg stattgefunden haben (Dornberg 2013). Dort haben Menschen miteinander gesägt und es wurde eine Mathematisierung dieses Geschehens, dieser Leistungsvorgänge angestrebt. Eine naturgetreue Abbildung oder Simulation von Leistung bzw. von Gesundheit und Krankheit oder von Rehabilitation. Das war überaus wichtig, weil es am Ende des Zweiten Weltkriegs und danach viele Kriegsversehrte gab. Die Frage nach der Rehabilitation stand im Raum: Wie können die Leute wieder in den Arbeitsprozess integriert werden, die durch den Krieg Behinderungen erfahren haben. Bei diesen Experimenten haben sie auch Gesunde und Kranke miteinander sägen lassen. Es stellte sich heraus, dass z. B. Parkinson-Kranke, wenn sie mit einem Gesunden zusammen sägen, fast genau dieselbe Leistung erbringen können wie zwei Gesunde. Das heißt, dass man dort einen systemischen umweltbezogenen Ansatz von Gesundheit und Krankheit oder von Rehabilitation entwickelt hat und das auch rechnerisch oder kybernetisch zeigen bzw. darstellen konnte. Die Baumsäge der Arbeitsgruppe in Heidelberg war mit Sensoren verbunden, die diesen Akt des Sägens auch visualisieren konnten. So wurden die Leistung, aber auch kritische Punkte des Sägeprozesses über Diagramme sichtbar gemacht. Damit wurde ein biokybernetisches Feld aufgebaut, das eine Art „Ununterschiedenheitszone“ (Deleuze 2016) ermöglichte zwischen der naturwissenschaftlichen mathematischen Gestalt einerseits und der phänomenologischen Gestalt des Sägens, der Erfahrung, der Spürebene andererseits. Das Entstehen und die Dynamik des Gemeinsamen konnten durch den Anschluss an ein technisches System einerseits erforscht werden, und waren andererseits an Befragungen gekoppelt. Die Kranken und die Gesunden wurden befragt, Studierende haben ebenso gesägt und wurden auch befragt: Wie erlebt ihr dieses Experimentalsystem? Durch beide Ebenen, die technisch-mathematische und die erfahrungsbezogene, hat man dieses Gemeinsame, diese Emergenz bzw. gewisse Qualitäten im gemeinsamen Sägen - dass es ,fluppt“, oder ,wann läuft es besser?` - sichtbar gemacht. So konnte eine objektiviertere Ebene erreicht werden.

DF: Glaubst du, dass diese Art der diagrammatischen Umsetzung und Beschreibung tatsächlich das Phänomen, die Erklärung dieses Phänomens hervorgebracht hat? Oder war dies nicht ein Holzweg, weil man dachte, dass Ingenieure das Ding erklären können? Als wir die Sägeapparatur in Heidelberg gesehen haben, wurde uns ja schnell klar, dass die Sägeerfahrung mit der Maschine im Vergleich zur tatsächlichen Baumsäge bedenklich unterkomplex umgesetzt war, also nur eingeschränkte Freiheitsgrade hatte. Das improvisatorische 
Moment, ohne das der normale Sägevorgang gar nicht erst nicht in Gang kommt geschweige denn läuft, wurde bei der Heidelberger Apparatur weitgehend außer Acht gelassen. Die beiden Sägenden werden dort schon in einer ziemlichen Zwangshaltung förmlich festgestellt, in einen Apparat eingespannt.

Das Experimentalsystem haben wir ja bereits mehrfach nachgestellt - in interkulturellen Konstellationen mit ägyptischen und deutschen Studierenden mitten im winterlichen Schwarzwald ${ }^{11}$ oder mit Michaela Ott und Marie-Luise Angerer im Ratssaal in Konstanz. ${ }^{12}$ Alle stellen sich zunächst einmal die Frage: Wer nimmt die Säge zuerst in die Hand, wer bietet sie dem Anderen an, wie setzt man dann gemeinsam an? Findet man zunächst keine Spurrille an dem Baumstamm, setzt man das Ding wieder ab, versucht es schräg, nimmt Blickkontakt auf usw. Alles Improvisation bis hin zu der Frage, wie man zu einem gelungenen Ende kommt. Aber schon der Aushandlungsprozess, der die Säge überhaupt in Position bringt, bleibt bei der Heidelberger Vorrichtung ausgespart. Da steht das Ingenieurs-Paradigma im Vordergrund und das möchte XY-Koordinaten und standardisierte Funktionen erfassen, um den Ablauf in ein Koordinatensystem zu packen. Aber es geht bei diesen mathematischen Übersetzungen bekanntlich viel verloren.

MD: Wir haben uns intensiv mit der Frage des Embodiments und der Frage der Umweltbildungen beschäftigt, also mit diesen berühmten vier E's: Embodiment, Embeddedness, Extended mind, Enactivism (Fingerhut et al. 2013). Gleichzeitig war uns die Frage der medialen Bezüge wichtig, die sich da beobachten lassen. Letztlich ging es darum, wie man dann das, was Verkörperung, Interaktion z. B. von zwei Improvisationsmusikern oder die Interaktion von Zuschauern eines Videofilms mit dem Videofilm ausmacht, theoretisch oder metatheoretisch fassen kann. Die These war, dass die sensorischen Ableitungen und die Diagramme, die der Sägeprozess erzeugt, dass diese die Interaktion zweier Subjekte, deren Geschehnishaftes, das Handlungshafte, das Performative zeigen können. Paradigmatisch steht dafür bei Paul Christian und Viktor von Weizsäcker von der Heidelberger Arbeitsgruppe die Sinuskurve. Der Neurologe und der Internist gelten als Mitbegründer der Psychosomatik und der „Gestaltkreislehre“ (Dornberg 2013; Weizsäcker 1973). Sie haben tatsächlich

\footnotetext{
${ }^{11}$ Siehe Experiencing the Two Handed Trim Saw: Intercorporality, Hyperlocality and Migration. http://www.mbodyresearch.de/front_content.php?idcat=63\&lang=1. Zugegriffen: 20. März 2018.

${ }^{12}$ Das Ergebnis kann man sich unter http://metaspace.de/tmp/angerer_ott.mp3 anhören. Zugegriffen: 20. März 2018.
} 
in diesem genormten, von den Ingenieuren in eine Zwangshaltung gebrachten System zeigen können oder wollen, dass ein ,gelungener' Sägeakt sich in Form einer Sinuskurve zeigt, eines klaren Tons im Grunde genommen, einer reinen Schwingung. Das Gelingen von „Funktionen“ oder von kybernetischen „Funktionskreisen“ klingt auch bei dem Begriff der „,biologischen Symphonie“ in Jakob von Uexküll's Umweltlehre an (Uexküll 1920). Die Harmonie. Es geht darum, wie z. B. auch bei den Pendelexperimenten, die von Weizsäcker und Christian unternommen haben, die Überlegenheit der menschlichen, biologischen ,Maschinen' gegenüber den rein technischen zu zeigen. Also, dass eine Maschine ein Seil nicht so ,schön` zum Schwingen bringen kann, und so funktional wie ein Mensch oder zwei Menschen: Die biologisch-lebendige Sinuskurve des Sägens oder des Pendelns wird letztlich als schöner und auch funktionaler wahrgenommen bzw. performiert als (durch) die rein technischen Apparaturen.

DF: Die körperliche Wende in der Robotik kam erst in den 1980er Jahren. Bereits in den Pendelexperimenten von Christian und von v. Weizsäcker wird die Ununterscheidbarkeit - wo hört die Hand auf, wo fängt das Seil an - betont. Und das ist auch eine morphologische Ununterscheidbarkeit: Denn nicht nur das Seil, sondern auch die Hand hat eine bestimmte Geometrie und verfügt mit ihren Sehnen, Knochen, der Haut und den Muskeln über ganz bestimmte Elastizitäten und physische Eigenschaften. Das hat man in der frühen Robotik über Jahrzehnte hinweg völlig vernachlässigt und erst in den 1980er Jahren - dreißig Jahre nach Viktor v. Weizsäcker - wiederentdeckt. Robotik war immer auch Biokybernetik, zumindest dann, wenn es um Prothesen ging. Ingenieure haben also über Jahrzehnte hinweg in der Robotik diesen KI-Ansatz verfolgt und versucht, mit zentralen Steuerungseinheiten, Sensoren und Aktoren körperbasierte Maschinen zu konstruieren. Erst Rodney Brooks und Rolf Pfeifer haben in den 1980er und 1990er Jahren mit Gummi, mit Federn, mit Holz und hautähnlichen Materialien gearbeitet, die in sich schon eine organische Morphologie und Schwingungseigenschaft einbringen, und die sich vor allem einer detaillierten Berechenbarkeit entziehen und gleichzeitig eine wirklich biologische Komponente mit ins Spiel bringen. Oder besser gesagt eine semibiologische, weil es sich um kein lebendes, aber doch organisches Material handelt. Das hat die Robotik enorm beflügelt. Das Mechanische war in einer Sackgasse und ich habe den Eindruck, die Sackgasse besteht als Gefahr auch bei diesem Heidelberger Instrument.

MD: Nein, ich sehe das anders. In den Heidelberger Experimenten wurde ziemlich klar gezeigt, dass durch die Interaktionen von lebenden Systemen mit anderen lebenden Systemen, also z. B. von zwei Menschen, zumindest momenthaft ein „dritter Körper“, eine emergente Leistung ermöglicht wird, die eine neue 
Qualität gewinnt, die es vorher nicht gab. Das trifft auch auf unsere Projekte zu (Dornberg und Fetzner 2017; Fetzner und Dornberg 2015): Die Künstler, die über Kontinente hinweg Musik machen und über Skype improvisieren, da entsteht Emergenz: wie sie miteinander agieren und z. B. Musik machen.... Gerade das konnten die Heidelberger Experimente schon zeigen. Was uns aber zunehmend interessierte, ist genau das, was auch in der Robotik quasi zu einem neuen Paradigma wurde, nämlich dass das Lebendige selbst, ja das Fehlerhafte, das Organische, das Biegsame, die Abweichung in der Schwingung, eigentlich kein Fehler ist, der auszuschließen ist, sondern dass dieses Element selbst etwas Neues ermöglicht, was in den Gesamtprozess eingeht, ihn verändert bzw. als genau diesen erst möglich macht.

DF: Dabei handelt es sich um eine Art Quasi-Intelligenz. Bis dato dachte man, man brauche einen Riesenrechner, der sämtliche Sensordaten prozessiert und dann auf die Aktoren, beispielsweise Servomotoren, als Handlungsanweisung überträgt. Also Gleichgewichtszustände, Temperatur- und Feuchtigkeitsstände auslesen, alles, was es so an elektronischen Sensoren gibt, um die gewonnen Daten dann zentral zu prozessieren. So hat die Robotik den menschlichen Organismus über Jahrhunderte hinweg cartesianisch als Apparatur verstanden. ${ }^{13}$ Bis man eben erkannt hat, dass in einem bestimmten Material und dessen Eigenschaften bereits eine Art sinnlicher Erfahrung und vielleicht auch Intelligenz schlummert, sodass ich auch nur noch einen Bruchteil an Rechenkapazität brauche. Das wesentliche Verhalten wird über die Beschaffenheit eines Materials und dessen Umwelteinbettungen entschieden. Der Fisch im Wasser, der Vogel in der Luft, die Schnecke auf dem Erdboden über die schleimige Spur, d. h. diese Einbettungen und „Nähte“, die sich da ausbilden, haben eine Geschmeidigkeit, eine eigene Funktionalität. Diese müssen dann nicht mehr verstanden, berechnet oder ausgelesen werden. Sie sind vielmehr von selbst wirkmächtig. Wenn man so will, kann man auch das als eine Synthese aus Beuys und Bense verstehen.

MD: Im Beispiel der Baumsäge wäre das alte kybernetische Modell, das auf Berechenbarkeit abzielt, eine Baumsäge ohne schwingendes Sägeblatt, nur als eine starre Verbindung anzusehen. Aber in das schwingende Sägeblatt geht eine jahrhundertelange Tradition im Umgang mit dem Sägen ein, die handwerklich, historisch und sozial ist. Also das, was du Intelligenz nennst, ist da schon verbaut. Das Sägeblatt hat eine historische, eine soziale Morphologie. Das sind die-

\footnotetext{
${ }^{13}$ Diese Tradition kann man zurückverfolgen auf Wolfgang von Kempelen, Ernst Kapp u. v. a.
} 
jenigen kybernetischen Experimentalsysteme, die uns zunehmend interessierten. Der nächste Schritt in unseren Projekten war dann die Auseinandersetzung mit dem „Parasitären“, mit dem Parasiten, der Störung. ${ }^{14}$ Unsere Projekte haben sich vom Gelingen immer mehr auch zu den Phänomenen der Störung hin entwickelt. Da gab es etwa die improvisierte Performance „Voice via Violin“ zwischen dem Violinisten Harald Kimmig und dem Stimmkünstler Jan F. Kurth, bei der es zu einer Störung kam (Fetzner und Dornberg 2015). ${ }^{15}$ Die Skype-Verbindung ist abgebrochen und wir hatten alle Angst, dass das ganze Experimentalsystem scheitert. Doch es ist gelungen, die Verbindung wiederherzustellen - vielleicht durch das Vorhandensein eines Dritten Körpers oder von Protention und Retention, wie Husserl sagen würde. Und zwar in einer für die beteiligten Künstler nahezu unmerklichen Form, sodass sie davon gesprochen haben, nie getrennt gewesen zu sein. Das hatte durchaus etwas Magisches. Ich weiß noch, wie wir mit Ludwig Jäger über die Störung philosophiert und überlegt hatten, wie man dieses Phänomen konzeptualisieren kann (vgl. Jäger 2004). Wir haben uns mit moderner Entwicklungspsychologie beschäftigt, in der Störungen und deren Bearbeitung in der Interaktion zwischen Eltern und Kind oder zwischen Therapeut und Patient gar nicht als Fehler gewertet, sondern als das wahrscheinlich wirksamste Moment in der Etablierung von Beziehungen gesehen wird. Störungen sind allgegenwärtig, müssen dauernd bearbeitet werden. Die Fähigkeit zur Reparatur, zur Elastizität, wie du das eben am Material erklärt hast, begründet überhaupt die tiefe Verbindung zwischen Eltern und Kindern oder zwischen Therapeut und Patient. Es steht also gar nicht das Planbare oder ein vorab berechnetes Ergebnis im Vordergrund, als vielmehr der gelingende Umgang mit Störungen und Passungsprozessen.

DF: Bei den Skype-Performances haben wir mit ImprovisationskünstlerInnen gearbeitet, die in der Lage sind, nicht nur mit Fehlern umzugehen, sondern die den Fehler brauchen oder die Abweichung oder das Unerwartete, die Brechung als ästhetisches Moment, um überhaupt das Geschehen am Laufen zu halten und die dann irgendwann in eine Art von Sättigung oder zu einem Ende kommen. Wir haben uns in unseren Projekten aus diesem Grund auch mit dem Begriff der „Zwischenleiblichkeit“ des französischen Philosophen Merleau-Ponty (2003, 1986; vgl. Fetzner und Dornberg 2015) beschäftigt und gefragt, wie sich dieses

\footnotetext{
${ }^{14}$ Vgl. unser Projekt „BUZZ - Parasitäre Ökologien“ (Dornberg 2017; Dornberg und Fetzner 2017). Siehe http://parasite.metaspace.de. Zugegriffen: 8. Juni 2018.

${ }^{15}$ Vgl. Voice via Violin. http://vvv.metaspace.de. Zugegriffen: 8. Juni 2018.
} 
Phänomen zwischen Skype-Musikern oder verschiedenen Orten hinweg translokal oder medial, beispielsweise musikalisch entwickeln kann. Dann hat sich in die Zwischenleiblichkeit die Fehlfunktion oder der Fehler eingeschlichen. Das wurde über den Begriff des „Parasiten“ dann besser konzeptualisierbar und hat zu unserem Forschungsprojekt BUZZ geführt. ${ }^{16}$

MD: Der Begriff der Zwischenleiblichkeit, der vorrangig von Merleau-Ponty entwickelt worden ist, ist ein philosophisches Konzept, ein phänomenologisches Konzept, mit dem solche Phänomene untersucht werden. Es geht etwa darum, dass derjenige, der das Seil schwingt, zwischen seiner Hand und dem Seil nicht mehr genau unterscheiden kann. Das Seil wächst sozusagen in die Hand hinein und die Hand in das Seil. Oder das Beispiel von Merleau-Ponty mit dem Spazierstock, der Hand und dem Boden, bei dem die Überwindung der Natur-Geist-Dichotomie problematisiert wird, die im Bodily-Turn Jahrzehnte später wiederaufgenommen wird. Darüber hinaus haben uns Spannungen interessiert, bei denen ein nicht-identisches Moment hereinkommt. Das Seil beim Spielen mit dem Seil ist doch nicht die Hand und der Stock des Spaziergängers ist doch nicht der Boden. Wo oder wie wird das spürbar? Wenn der Spaziergänger stolpert, oder wenn er den Stock aus der Hand verliert, oder wenn das Seil losgelassen wird oder wenn die Säge eben nicht mehr berechenbar ist? Aus diesem Grund haben wir ja beim Workshop in Konstanz die Säge auch an einen Tonabnehmer angeschlossen, um das Unberechenbare der Säge/ des Sägens zu zeigen. Und die akustischen Phänomene korrespondierten zum Teil damit, dass die Säge eine ganz eigene Art von Geräuschgenerator wird. Dadurch sollten Übergangs- und Hybridisierungsphänomene zwischen Mensch-Säge-Holz-Geräusch-Tonabnehmer-Tonabgeber usf. medial dargestellt und erlebbar gemacht werden. Parasitierungsprozesse, Unabgeschlossenes.

DF: Das ist interessant: Du sagst, wir haben die Säge an einen Tonabnehmer angeschlossen. Ich dachte jetzt eher, der Tonabnehmer wurde an die Säge angeschlossen. Nun kann man sagen, das sei egal, aber das ist es eigentlich nicht. Der Perspektivwechsel macht einen Unterschied. Der Tonabnehmer ändert durch seine Positionierung an der Säge auch das Sägeverhalten. Dadurch, dass die Beteiligten über diese artifizielle, prozessuale Verzerrung das Sägen (akustisch anders) hören, ändert sich auch ihr Sägevorgang. Damit haben wir das Baumsäge-Modell von Christian verkompliziert, technischer gemacht. Uns interessiert, was Luhmann „Beobachtungen zweiter Ordnung“ oder „Kybernetik

\footnotetext{
${ }^{16}$ BUZZ. Parasitic Ecologies. http://parasite.metaspace.de. Zugegriffen: 8. Juni 2018.
} 
zweiter Ordnung“ nennt, dass der Beobachter immer in das Beobachten eingeht, natürlich auch in die Medien und die Technik und dass man nie eine artefakt- oder technik- oder beobachtungsfreie Kybernetik, Bio-Kybernetik, Lebensform findet.

Das hat Bense in seiner „Technischen Existenz" sehr überzeugend dargelegt. Benses kybernetisches Referenz-Paradigma war die Heisenberg'sche Unschärferelation, die ja bekanntlich besagt, dass der Beobachter immer in das Beobachtete eingeht. Das kann man aber auch anders denken, nämlich dass die Phänomene von Teilchen und Welle den Beobachter selbst formieren und instruieren und ihn erst zu solchen Beobachtungen zweiter Ordnung befähigen.

Es geht dabei um die Infragestellung des Menschlichen als Paradigma. Darum, in einem posthumanen Beobachtungssystem den Beobachter selber technischer werden zu lassen, ihn zu einem Cyborg zu machen. Aber auch das wird wieder verwoben, weil der Denkfehler gerade wäre, dass dieser Cyborg eben als nicht menschlich gedacht wird. Bei unserem aktuellen Projekt über das Cochlea-Implantat ${ }^{17}$ wird genau diese Frage aufgeworfen. Der Denkfehler vieler Cyborg-Anhänger der ersten Generation war ja, dass man durch ein Implantat zu einem Roboter im schlechten Sinne wird.

Aber die Geschichte hat uns gezeigt, dass Roboter inzwischen viel biologischer, viel menschlicher, viel materialhafter, elastischer geworden sind. In ihnen ist immer ein Element von Parasitierung und von Störung enthalten: Material und Technik, Menschliches und Dingliches beeinflussen einander auf vielfältigste Weisen. Der Beobachter und das Beobachtete werden in parasitärer Weise befallen. Wir werden vom Material oder auch von anderen Lebewesen, mit denen wir interagieren, fortlaufend beeinflusst, parasitiert. Wir können aber auch lernen. Das hat uns bei unserer Arbeit dann immer mehr in die Richtung des Parasiten oder in Verbindung mit dem Tier gebracht, weil gerade das Mensch-Tier-Verhältnis und das Mensch-Technik-Verhältnis viele Parallelen aufweist. Denn es geht in beiden Fällen um eine wesenhafte existenzielle Auseinandersetzung mit dem ganz Anderen. Bis hin nicht nur zum Tier, sondern auch zu den Pflanzen. Und wenn man Uexküll mit seinem Begriff der Umwelt nimmt, kann man den natürlich beliebig fein auflösen: In jeder Umwelt gibt es viele andere Verflechtungen mit wieder anderen Umwelten und anderen Materialien. Die Umwelt kann ich z. B. in Sauerstoff und nach verschiedenen Materialien hin auflösen, welche alle mit unterschiedlichen Systemen menschlicher, tierischer ${ }^{17}$ Innovative Potenziale des Hörens mit dem Cochlea Implantat. http://ipoci.de. Zugegriffen:
8. Juni 2018. 
und dinglicher Aktanten verbunden sind. Das ergibt dann den Weg von dem Parasitären und Tierischen in unserem Projekt „BUZZ“18 zu unserem im Moment letzten Projekt „WASTELAND“ (Dornberg und Fetzner 2017),${ }^{19}$ in dem es um Müll geht, um Stoffwechselprodukte, also materielle Prozesse: Metabolismen, die in unseren Körper hinein, und aus dem Körper heraus, in unser Denken hinein, und aus dem Denken heraus wirken. Um Materie in einem weiten Sinn.

MD: Bei der Baumsäge und den darauf bezogenen Projekten haben uns deren materielle, elastische, störungsbezogene Aktanten, die sozusagen die Gelingungsorientierung im Sägen ein bisschen konterkarieren, über den Parasiten zu den Tieren, aber auch zu den Dingen und Materialien gebracht (Dornberg 2017). Und zur „objektorientierten Philosophie“ von Graham Harman (2016), gerade bei diesem Müll-Projekt, dem Ding-Projekt. Harmans Realismus ist ja „spekulativ“. Man darf das nicht unterschätzen, dass er keinesfalls behauptet, dass Objekte jetzt neuerdings ontologisch Subjekte seien, wie menschliche Wesen. Sondern es ist eine Spekulation, also: „lass uns einfach mal so tun, als ob Alles ein Objekt ist“ oder ganz komplexe Objekte sind. Als ein Gedankenexperiment.

DF: Harman geht es nur um einen anderen Einstiegspunkt im Denken. Mit Latour (2008) kann man sagen, dass die Unterscheidung zwischen menschlich und nicht-menschlich durchaus sinnvoll ist, die Subjekt-Objekt-Unterscheidung jedoch nicht. Letztere ist nicht mehr zeitgemäß oder sie ist eine Sackgasse der Moderne.

MD: Meiner Einschätzung nach geht es tatsächlich mehr um das Spekulative und damit auch um das Künstlerische. So wie Harman sagt, möglicherweise ist so eine Säge ein Objekt, genau wie die Menschen oder der Tonabnehmer. Sie hat in ihrer Materialität und Historizität eine ganz , bestimmte ' Textur, eine ganz ,bestimmte' Semiotik im guten Sinne, eine ,bestimmte' Elastizität und dieses Objekt schließt mal mehr, mal weniger an Menschen an, die ebenso ihre Vorerfahrungen mitbringen, die in einem ganz bestimmten System zusammen sägen oder musizieren. Diese Menschen sind in ihrer Textur selber komplexe, Objekte'. Das versuchen wir zu reflektieren, wenn wir spekulativ, künstlerisch denken, performieren oder installieren. Was passiert denn da? Und zwar als ein neues (spekulatives) Objekt, als transmediales Experimentalsystem, u. a. als Filme, Installationen und interaktive Dokumentationen und nicht nur als reine Theorie. Als Material.

\footnotetext{
${ }^{18}$ BUZZ. Parasitic Ecologies. http://parasite.metaspace.de. Zugegriffen: 8. Juni 2018.

${ }^{19}$ Siehe WASTELAND. http://waste.metaspace.de. Zugegriffen: 8. Juni 2018.
} 
DF: Wir gehen oft spekulativ vor, wenn wir Systeme aufsetzen oder Projekte angehen, die sowieso schwer planbar sind. Oft können wir gerade einmal im Nachhinein noch rekonstruieren, wie das Eine in das Andere übergeht oder überging. Daher rührt auch unsere Sympathie für die „Nordwest-Passagen“-Metapher bei Michel Serres (1994) als hodologische Suchbewegung, die sich der Komplexität der Welt mit der Bescheidenheit einer Ameise nähert. Oder wie Latour in seiner Ausstellung „Reset Modernity“ so schön deutlich gemacht hat: das Globale gibt es gar nicht bzw. nur als verführerische Perspektive einer suggerierten Ganzheit. Das Globale gibt es nur aus der Sicht des Schöpfers oder der Sicht Gottes (Latour 2016). Für uns Menschen kann es das naturgemäß nicht geben, weil wir teilnehmende Beobachter sind und verflochten in unsere nachbarschaftlichen Verhältnisse. Ein Irrtum der Moderne bestand ja genau darin, das Globale wörtlich zu nehmen mit der daraus resultierenden Hybris. Diese Kritik führen wir ja in unserem Projekt DE $\backslash$ GLOBALIZE $^{20}$ (2018-2020) auf der Suche nach dem Terrestrischen (Latour 2018) und der sogenannten „Critical Zone“21 fort.

MD: Das ist mir jetzt noch einmal klargeworden, dass das Experimentalsystem Baumsäge naturgemäß bescheiden sein muss. Da ist ja die Scheide/Entscheidung drin, das ist die Grenze. Und deshalb haben wir dieses Bezugssystem zum Teil überschritten. Aber wie alles Gute im Leben begleitet es einen mal mehr, mal weniger. Diese Projekte werden mich jedenfalls immer begleiten. Also in der Ärzte-Ausbildung, Therapeuten-Ausbildung sägen wir immer noch mit den Auszubildenden, und die ganz neuen Forschungen zur Sprachentwicklung zeigen, dass die Baumsäge als Experimentalsystem geeignet ist, zu zeigen, wie Sprache gebildet wird, oder wie Emotionen reguliert werden, dass das alles interaktive Prozesse sind, die Emergenzen, die ,[d]ritte Körper“ einerseits schaffen und andererseits brauchen. Die aber natürlich diese sofort auch wieder parasitieren, zersetzen, verändern. Dass ein Element des Gelingens, aber auch ein Element der Störung immer dabei ist.

Immer geht es uns dabei auch um den Bezug zur Medientheorie, dass es immer um das Dazwischenliegende, Mediale geht, um das Vermittelnde und zugleich Verändernde (Dornberg 2013).

Da wären wir wieder beim Objekthaften, Parasitären angekommen. Dass eben jede Improvisation auch ein Ende finden muss und dieses Ende auch die

\footnotetext{
${ }^{20}$ http://deglobalize.com. Zugegriffen: 8. Juni 2018.

${ }^{21}$ https://en.wikipedia.org/wiki/Earth\%27s_critical_zone. Zugegriffen: 8. Juni 2018.
} 
Improvisation erst zu dem macht, was sie gewesen sein wird. Eben ein harter Fakt. Jede Wespenkolonie muss irgendwann auch mal absterben oder einen neuen Ort finden und dann nochmal neu ansetzen. Dieses Element, was eben in der reinen Gelingensorientierung oder in der rein biokybernetischen Ordnung der früheren Art, alles ist planbar, der Strom fließt dauernd, so nicht aufgeht. Sondern der Strom fällt auch mal aus oder muss an- und ausgeschaltet werden. Es gibt eben keinen allumfassenden Kreislauf, den großen kybernetischen Kreislauf, wo alle Ströme immer fließen, sondern es gibt eben An und Aus und kleinere Ströme.

DF: In jedem Fall gibt es fortlaufend Spannungsschwankungen, im Digitalen wie im Analogen, nur dass man jetzt mit Schwellenwerten arbeitet. Die Lücken füllen sich mit parasitärem Rauschen.

\section{Literatur}

Bense, M. (1998). Technische Existenz. In M. Bense (Hrsg.), Ausgewählte Schriften (Bd. 3, S. 122-158). Stuttgart: Springer.

Bense, M. (1970). Existenzmitteilung aus San Franzisko. Stuttgart: Hake.

Christian, P. (1989). Anthropologische Medizin. Theoretische Pathologie und Klinik psychosomatischer Krankheitsbilder. Heidelberg: Springer.

Christian, P., \& Haas, R. (1949). Wesen und Formen der Bipersonalität. Enke: Springer.

Deleuze, G. (2000). Die Falte Leibnitz, und der Barock. Frankfurt a. M.: Suhrkamp.

Deleuze, G. (2016). Francis Bacon. Logik der Sensation. München: Fink. (Erstveröffentlichung 1981).

Deleuze, G., \& Guattari, F. (1992). Tausend Plateaus. Berlin: Merve. (Erstveröffentlichung 1980).

Dornberg, M. (2013). Die zweigriffige Baumsäge. Überlegungen zu Zwischenleiblichkeit, Umweltbezogenheit und Überpersonalität. In T. Breyer (Hrsg.), Grenzen der Empathie. Philosophische, psychologische und anthropologische Perspektiven (S. 239-259). Paderborn: Fink.

Dornberg, M. (2014). Dritte Körper. Leib und Bedeutungskonstitution in Psychosomatik und Phänomenologie. In A. Böhler, C. Herzog, \& A. Pechriggl (Hrsg.), Korporale Performanz. Zur bedeutungsgenerierenden Dimension des Leibes (S. 103-122). Bielefeld: transcript.

Dornberg, M. (2017). Mitgeteilte und parasitäre Emergenz. Zwei Modelle verkörpernder Evolution. In G. Etzelmüller, T. Fuchs, \& C. Tewes (Hrsg.), Verkörperung. Eine neue interdisziplinäre Anthropologie (S. 281-311). München: De Gruyter.

Dornberg, M., \& Fetzner, D. (2017). Experimentelle Taktilität. Zur medienökologischen Erforschung von Zwischenkörpern. In K. Harasser (Hrsg.), Auf Tuchfühlung Zur Medialität des Taktilen (S. 39-63). Frankfurt a. M.: Campus Verlag.

Fetzner, D. (2009). Von der Röhrentechnik des Rasterkörpers zur Existenzmitteilung aus San Franzisko. Medienexploration zum Körpergedächtnis von Max Bense. Hannover: Telepolis. 
Fetzner, D. (2017). Wild topologies in $360^{\circ}$. In A. Miles (Hrsg.), The material turn and Interactive Documentary. Zenodo. http://doi.org/10.5281/zenodo.1120448. Zugegriffen: 20. März 2018.

Fetzner, D. (2018). Zur Autonomie in parasitären Verhältnissen. In T. Brey-Mayländer (Hrsg.), Das Streben nach Autonomie (S. 81-122). Baden-Baden: Nomos.

Fetzner, D., \& Dornberg, M. (Hrsg.). (2015). Intercorporeal Splits. Künstlerische Forschung zur Medialität von Stimme, Haut, Rhythmus. Leipzig: Open House.

Fingerhut, J., Hufendiek, R., \& Wild, M. (Hrsg.). (2013). Philosophie der Verkörperung. Grundlagentexte zu einer aktuellen Diskussion. Berlin: Suhrkamp.

Harman, G. (2016). Immaterialism, objects and social theory. Cambridge: Graham Harman.

Jäger, L. (2004). Störung und Transparenz. Skizze zur performativen Logik des Medialen. In S. Krämer (Hrsg.), Performativität und Medialität (S. 35-74). München: Fink.

Latour, B. (2008). Wir sind nie modern gewesen. Frankfurt a. M.: Suhrkamp. (Erstveröffentlichung 1991).

Latour, B. (2016). Reset Modernity! Cambridge: Graham Harman.

Latour, B. (2018). Das terrestrische Manifest. Suhrkamp: Frankfurt.

Merleau-Ponty, M. (1986). Das Sichtbare und das Unsichtbare. München: Fink. (Erstveröffentlichung 1964).

Merleau-Ponty, M. (2003). Phänomenologie der Wahrnehmung. Berlin: De Gruyter. (Erstveröffentlichung 1945).

Rheinberger, H.-J. (2001). Experimentalsysteme und epistemische Dinge. Göttingen: Suhrkamp.

Rieger, S. (2003). Kybernetische Anthropologie. Frankfurt a. M.: Suhrkamp.

Serres, M. (1994). Die Nordwest-Passage. Berlin: Merve.

Snow, C. P. (1957). The two cultures. Cambridge: Graham Harman.

Theweleit, K. (2007). Übertragung, Gegenübertragung, Dritter Körper: Zur Gehirnveränderung durch neue Medien. Köln: Walther König.

von Uexküll, J. (1920). Theoretische Biologie. Berlin: De Gruyter.

von Uexküll, T., \& Wesiack, W. (1998). Theorie der Humanmedizin. München: Urban \& Schwarzenberg.

von Weizsäcker, V. (1973). Der Gestaltkreis. Theorie der Einheit von Wahrnehmen und Bewegen. Frankfurt a. M.: Suhrkamp.

Martin Dornberg ist Psychosomatiker und Philosoph. Er leitet das Zentrum für Psychosomatische Medizin und Psychotherapie im Ärztehaus am St. Josefskrankenhaus Freiburg und ist Lehrbeauftragter für Philosophie und für interdisziplinäre Anthropologie an der Albert-Ludwigs-Universität Freiburg. Zu seinen Forschungs- und Tätigkeitsschwerpunkten gehören Psychosomatik, Philosophien der Neuzeit/(Post-)Moderne, Theorien des Körpers und der Medien. Er ist Gründungsmitglied von ,mbody. Künstlerische Forschung in Medien, Somatik, Tanz und Philosophie“ (2008) und an philosophisch-künstlerischen Projekten u. a. mit Daniel Fetzner und am Theater Freiburg beteiligt. Er ist Co-Autor von „Intercorporeal Splits. Künstlerische Forschung zur Medialität von Stimme, Haut, Rhythmus (zus. mit D. Fetzner, 2015)“. Zu seinen Publikationen gehören u. a. Mitgeteilte und 
parasitäre Emergenz. Zwei Modelle verkörpernder Evolution. In: Verkörperung. Eine neue interdisziplinäre Anthropologie (hrsg. von G. Etzelmüller, T. Fuchs \& C. Tewes, 2017) und Experimentelle Taktilität. Zur medienökologischen Erforschung von Zwischenkörpern (zus. mit D. Fetzner). In Auf Tuchfühlung. Zur Medialität des Taktilen (hrsg. von K. Harrasser, 2017).

Daniel Fetzner ist Professor für Künstlerische Forschung an der Hochschule Offenburg und Lehrbeauftragter für Medienethnografie an der Albert-Ludwigs-Universität Freiburg. Als Medienkünstler ist er an zahlreichen Projekten beteiligt und betreibt künstlerische Forschung zur Phänomenologie der Medien in translokalen Räumen. Seine Forschungsschwerpunkte liegen in interaktiven Web-Dokumentationen, Medienökologien, sozialen Insekten und Parasiten sowie der Phänomenologie des Körpers. Er ist Autor von Von der Röhrentechnik des Rasterkörpers zur Existenzmitteilung aus San Franzisko. Medienexploration zum Körpergedächtnis von Max Bense (2009) und Intercorporeal Splits. Künstlerische Forschung zur Medialität von Stimme, Haut, Rhythmus (zus. mit D. Fetzner, 2015). Aktuelle Veröffentlichungen sind Zur Autonomie in parasitären Verhältnissen. In Autonomie (hrsg. von T. Breyländer, 2018) und Wild Topologies in $360^{\circ}$. In The Material Turn and Interactive Documentary (hrsg. Von A. Miles, 2017). 